\title{
HIGH-ORDER DIFFERENCE SCHEMES FOR CONVECTION-DIFFUSION INTERFACE PROBLEMS
}

\author{
I.TR. ANGELOVA \\ Center of Applied Mathematics and Informatics, \\ University of Rousse, Rousse 7017, Bulgaria \\ E-mail: iangelova@ru.acad.bg
}

Received February 2, 2005; revised November 25, 2005

\begin{abstract}
On non-uniform mesh new high-order compact finite difference approximations of the solution and the flux to convection-diffusion interface problems in one-dimension are constructed and analyzed. Explicit formulas based on new Marchuk integral identities that give $O\left(h^{2}\right), O\left(h^{4}\right), \ldots$ accuracy are derived. New numerical integration quadrature procedures for computing three-point schemes of any prescribed order of accuracy are developed. Numerical experiments confirm the theoretical results.
\end{abstract}

Key words: high-order difference schemes, convection-diffusion interface problems, numerical integration quadrature

\section{Introduction}

Many physical problems involve the combination of convective and diffusive processes. They occur in the field where mathematical modelling is important such as physics, engineering and in particularly on fluid dynamics and transport problems. Many papers are describing numerical approximations, as well as the various techniques for analyzing and overcoming the difficulties that each numerical method presents, see $[6,9,10,11,13,14,15,16,17]$.

We are concerned with numerical solution methods for solving on nonuniform mesh one-dimensional convection diffusion equation with a source singularity in the domain. A number of applied problems exhibit local solution behaviour that require higher level resolution in one area of the domain than in other. To achieve effective numerical simulation of physical processes with local behaviour it is important to employ adapted discretization procedure with high local accuracy. 
The compact difference schemes $[2,3,4,5,7,8,12,13,14,15,16,17,18,19]$ atractive because they require a minimal stencil of the grid. In the present paper we implement the schemes derived on the base of new Marchuk integral identities for reaction problems in [2].

We consider the convection-diffusion equation

$$
L u:=-\left(a(x) u^{\prime}\right)^{\prime}+b(x) u^{\prime}+c(x) u=g(x) \text { on }(0,1)
$$

with the Dirichlet boundary conditions

$$
u(0)=u_{0}, \quad u(1)=u_{1} .
$$

Suppose that $a, b, c, g$ are piecewise continuous functions on $[0,1]$ which can have single discontinuity at $x=\alpha, 0<\alpha<1$. Then, if we assume that $u$ is continuous at $x=\alpha$, then a formal integration of (1) gives

$$
\begin{aligned}
& L u=g(x) \text { on }(0, \alpha) \cup(\alpha, 1), \\
& {[u]_{\alpha} \equiv u(\alpha+0)-u(\alpha-0)=0,} \\
& {\left[a u^{\prime}\right]_{\alpha}=0 .}
\end{aligned}
$$

We note that some results of this paper will require a greater smoothness of the solution, respectively of the data to the left and right of $x=\alpha$. It is also assumed that

$$
0<a_{0} \leq a(x) \leq a_{1}, \quad|b(x)| \leq b_{1}, \quad 0 \leq c(x) \leq c_{1}, 0 \leq x \leq 1 .
$$

Let $Q_{\alpha}^{k}$ be the set of continuous functions $v \in Q_{\alpha}^{k}$, that are defined on $[0,1]$ and have piecewise derivatives up to order $k$, where $k$ is integer. The function $v$ and its derivatives can have bounded discontinuities only at the point $\alpha$. Further, we assume that the solution of problem (1.1)-(1.2) (respectively (1.3)-(1.5), (1.2)) belongs to $Q_{\alpha}^{k}$, with appropriate $k$.

This paper is arranged as follows. In Section 2, we present the new Marchuk integral identities and derive the difference schemes. In Section 3 we propose new quadrature formulas for numerical integration of the integrals that arise at the construction of the difference schemes. Numerical experiments are conducted to show that the proposed difference schemes have the predicted in the theory accuracy.

\section{High-order Difference Schemes Based on New Marchuk Integral Identities}

Let

$$
d(x)=\exp \left(\int_{x}^{\alpha} \frac{b(t)}{a(t)} d t\right)
$$


Then equation (1.1) can be written as a reaction-diffusion one:

$$
L u:=-\left(p(x) u^{\prime}\right)^{\prime}+q(x) u=f(x),
$$

where

$$
p(x)=a(x) d(x), q(x)=c(x) d(x), f(x)=g(x) d(x) .
$$

We rewrite the problem (2.2), (1.2) as follows:

$$
\left\{\begin{array}{l}
-w^{\prime}+q(x) u=f(x), x \in(0, \alpha) \cup(\alpha, 1) \\
u(0)=u_{0}, u(1)=u_{1} \\
{[u]_{\alpha}=0,[w]_{\alpha}=0}
\end{array}\right.
$$

where $w(x)=p(x) u^{\prime}(x)$ is the flux.

Let us consider on $[0,1]$ two systems of mesh points:

$$
\begin{array}{r}
\omega_{1 h}=\left\{x_{0}=0, x_{i}=x_{i-1}+h_{i}, h_{i}>0, i=1, \ldots, N, x_{N}=1\right\}, \\
\omega_{2 h}=\left\{x_{i+1 / 2}=\frac{x_{i}+x_{i+1}}{2}, i=0, \ldots, N-1,\right. \\
\left.\hbar_{1}=\frac{h_{1}}{2}, \quad \hbar_{i}=\frac{h_{i}+h_{i+1}}{2}, \hbar_{N}=\frac{h_{N}}{2}\right\},
\end{array}
$$

where $\alpha=x_{j}, 0<j<N$. Let

$$
\xi_{j}^{(0)}(x)=\left\{\begin{array}{l}
1, x \in\left[x_{j-1}, x_{j+1}\right], \\
0, x \notin\left[x_{j-1}, x_{j+1}\right],
\end{array} \quad \xi_{j}^{(1)}(x)= \begin{cases}\int_{x_{j-1}}^{x} \frac{\xi_{j}^{(0)}(t)}{p(t)} d t, x \in\left(x_{j-1}, x_{j}\right), \\
x_{j+1} \frac{\xi_{j}^{(0)}(t)}{p(t)} d t, x \in\left(x_{j}, x_{j+1}\right), \\
0, & x \notin\left[x_{j-1}, x_{j+1}\right] .\end{cases}\right.
$$

For $n \geq 1$ we introduce the following functions:

$$
\begin{aligned}
& \xi_{j}^{(2 n)}(x)=\left\{\begin{array}{l}
\int_{x_{j}-1}^{x} q(t) \xi_{j}^{(2 n-1)}(t) d t, x \in\left(x_{j-1}, x_{j}\right), \\
\int_{x}^{x_{j+1}} q(t) \xi_{j}^{(2 n-1)}(t) d t, x \in\left(x_{j}, x_{j+1}\right), \\
0, \quad x \notin\left[x_{j-1}, x_{j+1}\right],
\end{array}\right. \\
& \xi_{j}^{(2 n+1)}(x)=\left\{\begin{array}{l}
\int_{x_{j}-1}^{x} \frac{\xi_{j}^{(2 n)}(t)}{p(t)} d t, x \in\left(x_{j-1}, x_{j}\right), \\
\int_{x}^{x_{j+1}} \frac{\xi_{j}^{(2 n)}(t)}{p(t)} d t, x \in\left(x_{j}, x_{j+1}\right), \\
0, \quad x \notin\left[x_{j-1}, x_{j+1}\right] .
\end{array}\right.
\end{aligned}
$$


Let denote

$$
\psi_{j}^{(n)}(x)=\sum_{k=1}^{n} \xi_{j}^{(2 k-1)}(x), \quad n=1,2, \ldots
$$

Then applying the method developed in [2], we obtain the following Marchuk type identities:

$$
\begin{aligned}
& \frac{u_{j}-u_{j+1}}{\psi_{j}^{(n)}\left(x_{j}+0\right)}+\frac{u_{j}-u_{j-1}}{\psi_{j}^{(n)}\left(x_{j}-0\right)} \\
& +\left(\int_{x_{j-1}}^{x_{j}} q(x) \frac{\psi_{j}^{(n)}(x)}{\psi_{j}^{(n)}\left(x_{j}-0\right)} d x+\int_{x_{j}}^{x_{j+1}} q(x) \frac{\psi_{j}^{(n)}(x)}{\psi_{j}^{(n)}\left(x_{j}+0\right)} d x\right) u_{j} \\
& -\int_{x_{j-1}}^{x_{j}} w(x) \frac{\xi_{j}^{(2 n)}(x)}{p(x) \psi_{j}^{(n)}\left(x_{j}-0\right)} d x+\int_{x_{j}}^{x_{j+1}} w(x) \frac{\xi_{j}^{(2 n)}(x)}{p(x) \psi_{j}^{(n)}\left(x_{j}+0\right)} d x \\
& =\int_{x_{j-1}}^{x_{j}} f(x) \frac{\psi_{j}^{(n)}(x)}{\psi_{j}^{(n)}\left(x_{j}-0\right)} d x+\int_{x_{j}}^{x_{j+1}} f(x) \frac{\psi_{j}^{(n)}(x)}{\psi_{j}^{(n)}\left(x_{j}+0\right)} d x . \\
& \frac{u_{j}-u_{j+1}}{\psi_{j}^{(n)}\left(x_{j}+0\right)}+\frac{u_{j}-u_{j-1}}{\psi_{j}^{(n)}\left(x_{j}-0\right)} \\
& +\left(\int_{x_{j-1}}^{x_{j}} q(x) \frac{\psi_{j}^{(n-1)}(x)}{\psi_{j}^{(n)}\left(x_{j}-0\right)} d x+\int_{x_{j}}^{x_{j+1}} q(x) \frac{\psi_{j}^{(n-1)}(x)}{\psi_{j}^{(n)}\left(x_{j}+0\right)} d x\right) u_{j} \\
& +\int_{x_{j-1}}^{x_{j}} q(x) u(x) \frac{\xi_{j}^{(2 n-1)}(x)}{\psi_{j}^{(n)}\left(x_{j}-0\right)} d x+\int_{x_{j}}^{x_{j+1}} q(x) u(x) \frac{\xi_{j}^{(2 n-1)}(x)}{\psi_{j}^{(n)}\left(x_{j}+0\right)} d x \\
& =\int_{x_{j-1}}^{x_{j}} f(x) \frac{\psi_{j}^{(n)}(x)}{\psi_{j}^{(n)}\left(x_{j}-0\right)} d x+\int_{x_{j}}^{x_{j+1}} f(x) \frac{\psi_{j}^{(n)}(x)}{\psi_{j}^{(n)}\left(x_{j}+0\right)} d x .
\end{aligned}
$$

The integral identities (2.5), (2.6) can be used for derivation of high-order difference schemes. The main problem is how to approximate the integrals $\xi_{j}^{(k)}(x)$.

Similar identities can be obtained for the flux $w$, which satisfies the following problem 


$$
\left\{\begin{array}{l}
-z^{\prime}+\widetilde{q}(x) w=\widetilde{f}(x), x \in(0, \alpha) \cup(\alpha, 1), \\
{[w]_{\alpha}=0,[z]_{\alpha}=-\left[\frac{f(x)}{q(x)}\right]_{\alpha},} \\
w^{\prime}(0)=q(0) u_{0}-f(0), w^{\prime}(1)=q(1) u_{1}-f(1),
\end{array}\right.
$$

where

$$
\widetilde{p}(x)=\frac{1}{q(x)}, \widetilde{q}(x)=\frac{1}{p(x)}, \widetilde{f}(x)=\left(\frac{f(x)}{q(x)}\right)^{\prime}, z(x)=\widetilde{p}(x) w^{\prime}(x) .
$$

In addition to (1.6), we assume

$$
0<q_{0} \leq q(x) \leq q_{1} .
$$

The first two equations (2.7) are equivalent to the following one

$$
-\left(\widetilde{p}(x) w^{\prime}\right)^{\prime}+\widetilde{q}(x) w=\widetilde{f}(x)-\delta(x-\alpha)\left[\frac{f(x)}{p(x)}\right]_{\alpha},
$$

where $\delta(\cdot)$ is the Dirac delta function. Now, following [2] we have

$$
\begin{aligned}
& \frac{w_{j}-w_{j+1}}{\widetilde{\psi}_{j}^{(n)}\left(x_{j}+0\right)}+\frac{w_{j}-w_{j-1}}{\widetilde{\psi}_{j}^{(n)}\left(x_{j}-0\right)} \\
& +\left(\int_{x_{j-1}}^{x_{j}} \widetilde{q}(x) \frac{\widetilde{\psi}_{j}^{(n)}(x)}{\widetilde{\psi}_{j}^{(n)}\left(x_{j}-0\right)} d x+\int_{x_{j}}^{x_{j+1}} \widetilde{q}(x) \frac{\widetilde{\psi}_{j}^{(n)}(x)}{\widetilde{\psi}_{j}^{(n)}\left(x_{j}+0\right)} d x\right) w_{j} \\
& -\int_{x_{j-1}}^{x_{j}} z(x) \frac{\widetilde{\xi}_{j}^{(2 n)}(x)}{\widetilde{p}(x) \widetilde{\psi}_{j}^{(n)}\left(x_{j}-0\right)} d x+\int_{x_{j}}^{x_{j+1}} z(x) \frac{\widetilde{\xi}_{j}^{(2 n)}(x)}{\widetilde{p}(x) \widetilde{\psi}_{j}^{(n)}\left(x_{j}+0\right)} d x \\
& =\int_{x_{j-1}}^{x_{j}} \widetilde{f}(x) \frac{\widetilde{\psi}_{j}^{(n)}(x)}{\widetilde{\psi}_{j}^{(n)}\left(x_{j}-0\right)} d x+\int_{x_{j}}^{x_{j+1}} \widetilde{f}(x) \frac{\widetilde{\psi}_{j}^{(n)}(x)}{\widetilde{\psi}_{j}^{(n)}\left(x_{j}+0\right)} d x-\left[\frac{f(x)}{q(x)}\right]_{\alpha} .
\end{aligned}
$$

The integral identity corresponding to the left boundary condition is:

$$
\begin{aligned}
& \frac{w_{0}-w_{1}}{\widetilde{\psi}_{0}^{(n)}(0+)}+w_{0} \int_{0}^{x_{1}} \widetilde{q}(x) \frac{\widetilde{\psi}_{0}^{(n)}(x)}{\widetilde{\psi}_{0}^{(n)}(0+)} d x+\int_{0}^{x_{1}} z(x) \frac{\widetilde{\xi}_{0}^{(2 n)}(x)}{\widetilde{p}(x) \widetilde{\psi}_{0}^{(n)}(0+)} d x \\
& =\int_{0}^{x_{1}} \widetilde{f}(x) \frac{\widetilde{\psi}_{0}^{(n)}(x)}{\widetilde{\psi}_{0}^{(n)}(0+)} d x-z_{0},
\end{aligned}
$$

where $z_{0}=u_{0}-f(0) / q(0)$,

$$
\widetilde{\xi}_{0}^{(0)}(x)=\left\{\begin{array}{ll}
1, & x \in\left[0, x_{1}\right), \\
0, & x \in\left[x_{1}, 1\right],
\end{array} \widetilde{\xi}_{0}^{(2 n-1)}(x)= \begin{cases}\int_{x}^{x_{1}} \frac{\widetilde{\xi}_{0}^{(2 n-2)}(t)}{\tilde{p}(t)} d t, & x \in\left[0, x_{1}\right), \\
0, & x \in\left[x_{1}, 1\right],\end{cases}\right.
$$




$$
\widetilde{\xi}_{0}^{(2 n)}(x)=\left\{\begin{array}{ll}
\int_{x}^{x_{1}} \widetilde{q}(t) \widetilde{\xi}_{0}^{(2 n-1)} d t, & , x \in\left[0, x_{1}\right), \\
0 & , x \in\left[x_{1}, 1\right],
\end{array} \widetilde{\psi}_{j}^{(n)}(x)=\sum_{k=1}^{n} \widetilde{\xi}_{j}^{(2 k-1)}(x),\right.
$$

for $n=1,2, \ldots$

Let $U$ denote the discrete function approximating the differential solution. Then, if we ignore the integral containing the flux $w$, we will obtain the scheme:

$$
\left\{\begin{array}{l}
-a_{j}^{(n)} U_{j-1}+c_{j}^{(n)} U_{j}-b_{j}^{(n)} U_{j+1}=f_{j}^{(n)}, j=1, \ldots, N-1, \\
U_{0}=u_{0}, \quad U_{N}=u_{1}, \quad n=1,2, \ldots,
\end{array}\right.
$$

where

$$
\begin{aligned}
& a_{j}^{(n)}=\frac{1}{\hbar_{j} \psi_{j}^{(n)}\left(x_{j}-0\right)}, \quad b_{j}^{(n)}=\frac{1}{\hbar_{j} \psi_{j}^{(n)}\left(x_{j}+0\right)}, \\
& c_{j}^{(n)}=a_{j}^{(n)}+b_{j}^{(n)}+\frac{1}{\hbar_{j}}\left[\int_{x_{j-1}}^{x_{j}} \frac{q(x) \psi_{j}^{(n)}(x)}{\psi_{j}^{(n)}\left(x_{j}-0\right)} d x+\int_{x_{j}}^{x_{j+1}} \frac{q(x) \psi_{j}^{(n)}(x)}{\psi_{j}^{(n)}\left(x_{j}+0\right)} d x\right], \\
& f_{j}^{(n)}=\frac{1}{\hbar_{j}}\left[\int_{x_{j-1}}^{x_{j}} \frac{f(x) \psi_{j}^{(n)}(x)}{\psi_{j}^{(n)}\left(x_{j}-0\right)} d x+\int_{x_{j}}^{x_{j+1}} \frac{f(x) \psi_{j}^{(n)}(x)}{\psi_{j}^{(n)}\left(x_{j}+0\right)} d x\right] .
\end{aligned}
$$

In order to derive from (2.10)-(2.13) finite difference schemes up to fourth order of accuracy, classical numerical integration formulas (e.g. midpoint, trapezoid or Simpson's rule) can be used. For example, to obtain the popular "standard interface formula" proposed by Varga [20] (see also [16]) we apply the midpoint rule to $a_{j}^{(1)}, b_{j}^{(1)}$ and the trapezoid rule to the other integrals in $c_{j}^{(1)}$ and $f_{j}^{(1)}$ :

$$
\begin{aligned}
& a_{j}^{(1)}=\frac{p_{j-1 / 2}}{h_{j} \hbar_{j}}, \quad b_{j}^{(1)}=\frac{p_{j+1 / 2}}{h_{j+1} \hbar_{j}}, \quad c_{j}^{(1)}=a_{j}^{(1)}+b_{j}^{(1)}+\frac{h_{j} q_{j}^{-}+h_{j+1} q_{j}^{+}}{h_{j}+h_{j+1}}, \\
& f_{j}^{(1)}=\frac{h_{j} f_{j}^{-}+h_{j+1} f_{j}^{+}}{h_{j}+h_{j+1}} .
\end{aligned}
$$

In [2] Lobatto's formulas [3] were used for derivation of higher order numerical integrations. In the next section we present the new ones.

The following result holds for the rate of convergence of the scheme (2.10)(2.13).

Theorem 1. Let $a \in Q_{\alpha}^{2 n-1}, b, c g \in Q_{\alpha}^{2 n-2}, u$ be the solution of the differential problem (1.1), (1.2) and $U$ is the solution of the difference problem (2.10)-(2.13). Then

$$
\|u-U\|_{\infty} \leq C \hbar^{2 n},
$$

where the constant $C$ doesn't depend on $\hbar=\sqrt[2 n]{\sum_{k=1}^{N} h_{k}^{2 n+1}}$. 
The proof is very similar to that one of Theorem 2 given in [2] and we omit it.

\section{Numerical Integration}

\subsection{One-dimensional integral}

Let us consider the one-dimensional integral

$$
\xi^{1}:=\xi^{1}(f ; h)=\int_{0}^{h} f(x) d x=h \int_{0}^{1} f(h x) d x, \text { for } f(x) \in C^{n+1}[0, h] .
$$

We write $f(x)$ in Maclaurin series

$$
f(x)=\sum_{k=0}^{n} f_{k} x^{k}+\mathcal{O}\left(x^{n+1}\right), \quad \text { where } f_{k}=\frac{f^{(k)}(0)}{k !} .
$$

Then for $\xi^{1}$ we have

$$
\xi^{1}=h \sum_{k=0}^{n} f_{k} \frac{h^{k}}{k+1}+\mathcal{O}\left(h^{n+2}\right) .
$$

Let introduce the auxiliary function

$$
\begin{aligned}
T(\alpha) & \equiv \frac{1}{2}\left[f\left((1-\alpha) \frac{h}{2}\right)+f\left((1+\alpha) \frac{h}{2}\right)\right] \\
& =\sum_{k=0}^{n} f_{k} \frac{h^{k}}{2^{k}} \sum_{l=0}^{\left[\frac{k}{2}\right]}\left(\begin{array}{c}
k \\
2 l
\end{array}\right) \alpha^{2 l}+\mathcal{O}\left(h^{n+1}\right), \alpha \in[0,1] .
\end{aligned}
$$

We seek quadrature formulas for $\xi^{1}$ of the form:

$$
\begin{aligned}
\xi^{1} & =h \sum_{i=1}^{S} w_{i} T\left(\alpha_{i}\right)+\mathcal{O}\left(h^{n+2}\right) \\
& =h \sum_{k=0}^{n} f_{k} \frac{h^{k}}{2^{k}} \sum_{l=0}^{\left[\frac{k}{2}\right]}\left(\begin{array}{c}
k \\
2 l
\end{array}\right) \sum_{i=1}^{S} w_{i} \alpha_{i}^{2 l}+\mathcal{O}\left(h^{n+2}\right),
\end{aligned}
$$

where $w_{i}>0$ are unknown weights and $\alpha_{i}$ are unknown real numbers. Equalizing in (3.2), (3.4) the coefficients at the corresponding degrees of $h$, we find:

$$
\sum_{l=0}^{\left[\frac{k}{2}\right]}\left(\begin{array}{c}
k \\
2 l
\end{array}\right) \sum_{i=1}^{S} w_{i} \alpha_{i}^{2 l}=\frac{2^{k}}{k+1}, \quad k=0,1, \cdots, n .
$$

After some rearranging, we obtain the following system with respect to $w_{i}, \alpha_{i}, i=1, \ldots, S$ : 


$$
\sum_{i=1}^{S} w_{i} \alpha_{i}^{2 l}=\frac{1}{2 l+1}, \quad l=0,1, \ldots,\left[\frac{n}{2}\right], \quad S=1,2, \ldots
$$

The accuracy of the quadrature formula $(3.4)$ is of order $2\left[\frac{n}{2}\right]+3$.

1) Let $S=1$. Then (3.5) takes the form

$$
\begin{aligned}
& \mathcal{O}\left(h^{3}\right): \quad w_{1}=1, \quad l=0, \\
& \mathcal{O}\left(h^{5}\right): \quad w_{1} \alpha_{1}^{2}=\frac{1}{3}, \quad l=1 .
\end{aligned}
$$

If we did not use the second equation of the last system, i.e. taking $\alpha$ as a free parameter, we can derive some known classical formulas. For $w_{1}=$ $1, \alpha_{1}=0$ we get the midpoint rule:

$$
\xi^{1}=h T(0)+\mathcal{O}\left(h^{3}\right)=h f\left(\frac{h}{2}\right)+\mathcal{O}\left(h^{3}\right) .
$$

For $w_{1}=1, \alpha_{1}=1$ we get the trapezoid formula:

$$
\xi^{1}=h T(1)+\mathcal{O}\left(h^{3}\right)=\frac{h}{2}[f(0)+f(h)]+\mathcal{O}\left(h^{3}\right) .
$$

Solving the full system, we find $w_{1}=1, \alpha_{1}=\sqrt{\frac{1}{3}}$, which leads to a formula of Lobato's type:

$$
\xi^{1}=h T\left(\frac{1}{\sqrt{3}}\right)+\mathcal{O}\left(h^{5}\right)=\frac{h}{2}\left[f\left(\left(1-\frac{1}{\sqrt{3}}\right) \frac{h}{2}\right)+f\left(\left(1+\frac{1}{\sqrt{3}}\right) \frac{h}{2}\right)\right]+\mathcal{O}\left(h^{5}\right) .
$$

2) Let $S=2$. Then, it follows from the system (3.5), that:

$$
\begin{aligned}
& \mathcal{O}\left(h^{3}\right): \quad w_{1}+w_{2}=1, \quad l=0, \\
& \mathcal{O}\left(h^{5}\right): \quad w_{1} \alpha_{1}^{2}+w_{2} \alpha_{2}^{2}=\frac{1}{3}, \quad l=1, \\
& \mathcal{O}\left(h^{7}\right): \quad w_{1} \alpha_{1}^{4}+w_{2} \alpha_{2}^{4}=\frac{1}{5}, l=2, \\
& \mathcal{O}\left(h^{9}\right): \quad w_{1} \alpha_{1}^{6}+w_{2} \alpha_{2}^{6}=\frac{1}{7}, \quad l=3 .
\end{aligned}
$$

If we take $w_{1}=\frac{2}{3}, w_{2}=\frac{1}{3}, \alpha_{1}=0, \alpha_{2}=1$, then only the first and second equations are satisfied. The corresponding formula coincides with the Simpson's rule and it is of order $\mathcal{O}\left(h^{5}\right)$ :

$$
\xi^{1}=h\left[\frac{2}{3} T(0)+\frac{1}{3} T(1)\right]+\mathcal{O}\left(h^{5}\right)=\frac{h}{6}\left[f(0)+4 f\left(\frac{h}{2}\right)+f(h)\right]+\mathcal{O}\left(h^{5}\right)
$$

For $w_{1}=\frac{4}{9}, w_{2}=\frac{5}{9}, \alpha_{1}=0, \alpha_{2}=\sqrt{\frac{3}{5}}$ the first three equations are satisfied and we get the integration formula 


$$
\xi^{1}=\frac{h}{18}\left[5 f\left(\left(1-\sqrt{\frac{3}{5}}\right) \frac{h}{2}\right)+8 f\left(\frac{h}{2}\right)+5 f\left(\left(1+\sqrt{\frac{3}{5}}\right) \frac{h}{2}\right)\right]+\mathcal{O}\left(h^{7}\right) .
$$

For $w_{1}=\frac{5}{21}, w_{2}=\frac{16}{21}, \alpha_{1}=1, \alpha_{2}=\frac{\sqrt{2}}{4}$ again only the first three equations are satisfied:

$$
\xi^{1}=\frac{h}{42}\left[5(f(0)+f(h))+16\left(f\left(\left(1-\frac{\sqrt{2}}{4}\right) \frac{h}{2}\right)+f\left(\left(1+\frac{\sqrt{2}}{4}\right) \frac{h}{2}\right)\right)\right]+\mathcal{O}\left(h^{7}\right) .
$$

Now, for the unique solution of the full system

$w_{1}=\frac{1}{2}\left(1+\frac{1}{3} \sqrt{\frac{5}{6}}\right), w_{2}=\frac{1}{2}\left(1-\frac{1}{3} \sqrt{\frac{5}{6}}\right), \alpha_{1}^{2}=\frac{3}{7}-\frac{2}{7} \sqrt{\frac{6}{5}}, \alpha_{2}^{2}=\frac{3}{7}+\frac{2}{7} \sqrt{\frac{6}{5}}$

the corresponding formula is valid:

$$
\begin{aligned}
& \xi^{1}=\frac{h}{4}\left[\left(1+\frac{1}{3} \sqrt{\frac{5}{6}}\right)\left(f\left(\left(1-\sqrt{\frac{3}{7}-\frac{2}{7} \sqrt{\frac{6}{5}}}\right) \frac{h}{2}\right)+f\left(\left(1+\sqrt{\frac{3}{7}-\frac{2}{7} \sqrt{\frac{6}{5}}}\right) \frac{h}{2}\right)\right)\right. \\
& \left.+\left(1-\frac{1}{3} \sqrt{\frac{7}{6}}\right)\left(f\left(\left(1-\sqrt{\frac{3}{7}+\frac{2}{7} \sqrt{\frac{6}{5}}}\right) \frac{h}{2}\right)+f\left(\left(1+\sqrt{\frac{3}{7}-\frac{2}{7} \sqrt{\frac{6}{5}}}\right) \frac{h}{2}\right)\right)\right]+\mathcal{O}\left(h^{9}\right) .
\end{aligned}
$$

\subsection{Two-dimensional integral}

Let us consider the two-dimensional integral

$$
\xi^{2}:=\xi^{2}(f, g ; h)=\int_{0}^{h} f(x) d x \int_{0}^{x} g(y) d y=h^{2} \int_{0}^{1} f(h x) d x \int_{0}^{x} g(h y) d y,
$$

where $f \in C^{n+2}[0, h]$ and $g \in C^{n+1}[0, h]$. Now we expand $f$ and $g$ in Maclaurin's series:

$$
g(y)=\sum_{k=0}^{n} g_{k} y^{k}+\mathcal{O}\left(y^{n+1}\right), \quad f(x)=\sum_{k=0}^{n+1} f_{k} x^{k}+\mathcal{O}\left(x^{n+2}\right) .
$$

Then

$$
\xi^{2}=\frac{h^{2}}{2 !} \sum_{l=0}^{n} h^{l} \sum_{k=0}^{l} g_{k} f_{l-k} \frac{2 !}{(k+1)(l+2)}+\mathcal{O}\left(h^{n+3}\right) .
$$

Let us introduce the auxiliary function (see Fig.1):

$$
\begin{aligned}
T(\alpha) & =\frac{1}{3}\left[f\left(\frac{2 h}{3}(1-\alpha)\right) g\left(\frac{h}{3}(1-\alpha)\right)\right. \\
& \left.+f\left(\frac{2 h}{3}\left(1+\frac{\alpha}{2}\right)\right)\left(g\left(\frac{h}{3}(1-\alpha)\right)+g\left(\frac{h}{3}(1+2 \alpha)\right)\right)\right] .
\end{aligned}
$$




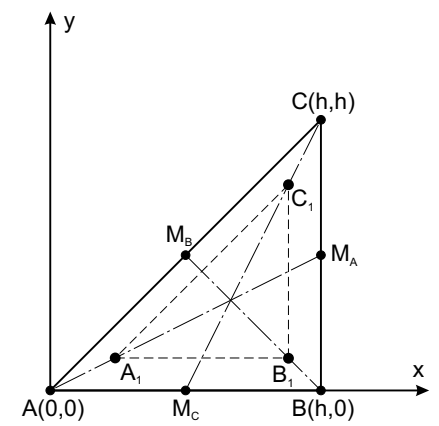

Figure 1. 2D-domain: $A_{1}\left(\frac{2 h}{3}(1-\alpha), \frac{h}{3}(1-\alpha)\right), B_{1}\left(\frac{2 h}{3}\left(1+\frac{\alpha}{2}\right), \frac{h}{3}(1-\alpha)\right), C_{1}\left(\frac{2 h}{3}(1+\right.$ $\left.\left.\frac{\alpha}{2}\right), \frac{h}{3}(1+2 \alpha)\right)$.

In view of (3.7), we get

$T(\alpha)=\sum_{l=0}^{n} \frac{h^{l}}{3^{l+1}} \sum_{k=0}^{l} g_{k} f_{l-k}\left[(1-\alpha)^{l} 2^{l-k}+(2+\alpha)^{l-k}\left((1-\alpha)^{k}+(1+2 \alpha)^{k}\right)\right]+\mathcal{O}\left(h^{n+1}\right)$.

We seek $w_{i}, \alpha_{i}$, such that:

$$
\xi^{2}=\frac{h^{2}}{2 !} \sum_{i=1}^{S} w_{i} T\left(\alpha_{i}\right)+\mathcal{O}\left(h^{n+3}\right) .
$$

After equalizing in (3.8)-(3.10) the coefficients at the corresponding powers of $h$ we get the system of nonlinear algebraic equations for $k=0,1, \ldots, l, s \geq$ 1 :

$$
\sum_{i=1}^{S} w_{i}\left[\left(1-\alpha_{i}\right)^{l} 2^{l-k}+\left(2+\alpha_{i}\right)^{l-k}\left(\left(1-\alpha_{i}\right)^{k}+\left(1+2 \alpha_{i}\right)^{k}\right)\right]=\frac{3^{l} 6}{(k+1)(l+2)} .
$$

From here, we find for $l \leq 4$ :

$$
\begin{array}{ll}
\mathcal{O}\left(h^{4}\right): & \sum_{i=1}^{S} w_{i}=1, \quad l=0,1, \\
\mathcal{O}\left(h^{5}\right): & \sum_{i=1}^{S} w_{i} \alpha_{i}^{2}=\frac{1}{4}, \quad l=2, \\
\mathcal{O}\left(h^{6}\right): & \sum_{i=1}^{S} w_{i} \alpha_{i}^{3}=\frac{1}{10}, \quad l=3, \\
\mathcal{O}\left(h^{7}\right): & \sum_{i=1}^{S} w_{i} \alpha_{i}^{4}=\frac{1}{10}, \quad l=4 .
\end{array}
$$

We are interested in formulas of seventh order of accuracy, which can be obtained for $S=3$. One possible solution is given by $w_{1}=\frac{3(9-\sqrt{21})}{160}$, 
$w_{2}=\frac{87+17 \sqrt{21}}{480}, w_{3}=\frac{39-\sqrt{21}}{60}, \alpha_{1}=0, \alpha_{2}=\frac{\sqrt{21}-1}{5}, \alpha_{3}=-\frac{\alpha_{2}}{2}$, then we get the following integration formula

$$
\begin{aligned}
\xi^{2}=\frac{h^{2}}{2}\left[\frac{3(9-\sqrt{21})}{160} T(0)\right. & +\frac{87+17 \sqrt{21}}{480} T\left(\frac{\sqrt{21}-1}{5}\right) \\
& \left.+\frac{39-\sqrt{21}}{60} T\left(\frac{1-\sqrt{21}}{10}\right)\right]+\mathcal{O}\left(h^{7}\right) .
\end{aligned}
$$

\subsection{Three-dimensional integral}

Let us consider the $3 D$ integral

$$
\begin{array}{r}
\xi^{3}:=\xi^{3}(f, g, r ; h)=\int_{0}^{h} f(x) d x \int_{0}^{x} g(t) d t \int_{0}^{t} r(y) d y \\
=h^{3} \int_{0}^{1} f(h x) d x \int_{0}^{x} g(h t) d t \int_{0}^{t} r(h y) d y
\end{array}
$$

where $f \in C^{n+3}[0, h], g \in C^{n+2}[0, h]$ and $r \in C^{n+1}[0, h]$.

We expand $f, g$ and $r$ in Maclaurin's series

$$
\begin{aligned}
& r(y)=\sum_{k=0}^{n} r_{k} y^{k}+\mathcal{O}\left(y^{n+1}\right), \quad g(t)=\sum_{k=0}^{n+1} g_{k} t^{k}+\mathcal{O}\left(t^{n+2}\right) \\
& f(x)=\sum_{k=0}^{n+2} f_{k} x^{k}+\mathcal{O}\left(x^{n+3}\right) .
\end{aligned}
$$

Then

$$
\xi^{3}=\frac{h^{3}}{3 !} \sum_{t=0}^{n} h^{t} \sum_{l=0}^{t} \sum_{k=0}^{l} r_{k} g_{l-k} f_{t-l} \frac{3 !}{(k+1)(l+2)(t+3)}+\mathcal{O}\left(h^{n+4}\right) .
$$

We introduce the function (see Fig.2):

$$
\begin{aligned}
T(\alpha)= & \frac{1}{4}\left[\left(f\left(\frac{3 h}{4}(1-\alpha)\right)+f\left(\frac{3 h}{4}\left(1+\frac{\alpha}{3}\right)\right)\right) g\left(\frac{h}{2}(1-\alpha)\right) r\left(\frac{h}{4}(1-\alpha)\right)\right. \\
& \left.+f\left(\frac{3 h}{4}\left(1+\frac{\alpha}{3}\right)\right) g\left(\frac{h}{2}(1+\alpha)\right)\left(r\left(\frac{h}{4}(1+\alpha)\right)+r\left(\frac{h}{4}(1+3 \alpha)\right)\right)\right] .
\end{aligned}
$$

We seek $w_{i}, \alpha_{i}$, such that: We seek $w_{i}, \alpha_{i}$, such that:

$$
\xi^{3}=\frac{h^{3}}{3 !} \sum_{i=1}^{S} w_{i} T\left(\alpha_{i}\right)+\mathcal{O}\left(h^{n+4}\right)
$$

In an analogical way as for $\xi^{1}, \xi^{2}$, we obtain: 


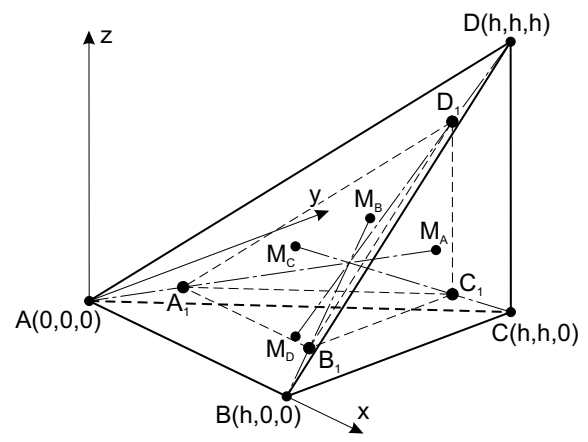

Figure 2. 3D-domain: $A_{1}\left(\frac{3 h}{4}(1-\alpha), \frac{h}{2}(1-\alpha), \frac{h}{4}(1-\alpha)\right), B_{1}\left(\frac{3 h}{4}\left(1+\frac{\alpha}{3}\right), \frac{h}{2}(1-\right.$ $\left.\alpha), \frac{h}{4}(1-\alpha)\right), C_{1}\left(\frac{3 h}{4}\left(1+\frac{\alpha}{3}\right), \frac{h}{2}(1+\alpha), \frac{h}{4}(1-\alpha)\right), D_{1}\left(\frac{3 h}{4}\left(1+\frac{\alpha}{3}\right), \frac{h}{2}(1+\alpha), \frac{h}{4}(1+3 \alpha)\right)$.

$$
\begin{array}{lll}
\mathcal{O}\left(h^{5}\right): & \sum_{i=1}^{S} w_{i}=1, & l=0,1, \\
\mathcal{O}\left(h^{6}\right): & \sum_{i=1}^{S} w_{i} \alpha_{i}^{2}=\frac{1}{5}, \quad l=2, \\
\mathcal{O}\left(h^{7}\right): & \sum_{i=1}^{S} w_{i} \alpha_{i}^{3}=\frac{1}{15}, \quad l=3 .
\end{array}
$$

Based on this system various formulas (up to the seventh order of accuracy) can be derived. We only present the following two formulas of order seven:

$$
\text { a) } \begin{aligned}
w_{1} & =-\frac{4}{5}, w_{2}=\frac{9}{5}, \alpha_{1}=0, \alpha_{2}=\frac{1}{3}, \\
\xi^{3} & =\frac{h^{3}}{6}\left[\frac{9}{5} T\left(\frac{1}{3}\right)-\frac{4}{5} T(0)\right]+\mathcal{O}\left(h^{7}\right), \\
\text { b) } w_{1} & =-\frac{1}{15}, w_{2}=\frac{16}{15}, \alpha_{1}=0, \alpha_{2}=\frac{1}{2}, \\
\xi^{3} & =\frac{h^{3}}{6}\left[\frac{16}{15} T\left(\frac{1}{2}\right)-\frac{1}{15} T(0)\right]+\mathcal{O}\left(h^{7}\right) .
\end{aligned}
$$

Following the same idea, one can derive formulas of numerical integration for the integrals $\xi_{j}^{(4)}, \xi_{j}^{(5)}, \xi_{j}^{(6)}, \cdots$. In the next section results of numerical experiments are given for difference schemes of order $2,4,6$.

\section{Numerical Experiments}

In this section results of numerical experiments illustrating the accuracy of the schemes derived in Section 3 are presented. Let $g(x)$ be chosen such that the problem (1.1), (1.2) with coefficients 


$$
\begin{aligned}
& a(x)=\left\{\begin{array}{l}
\exp (x-0.5), 0<x<0.75 \\
\exp (x), 0.75<x<1,
\end{array}\right. \\
& b(x)=\left\{\begin{array}{l}
b_{l}, 0<x<0.75, \\
b_{r}, 0.75<x<1,
\end{array} \quad c(x)= \begin{cases}\frac{1}{1+x}, 0<x<0.75 \\
\frac{2}{1+x}, 0.75<x<1,\end{cases} \right.
\end{aligned}
$$

has the exact solution

$$
u(x)=\left\{\begin{array}{l}
\exp (x), 0<x<0.75 \\
\exp (x-0.5)+\exp (0.75)-\exp (0.25), \quad 0.75<x<1
\end{array}\right.
$$

Table 1. Example: the solution $u, b_{l}=-1 ; b_{r}=1$.

\begin{tabular}{cccccccc}
\hline$N$ & 4 & 8 & 16 & 32 & 64 & 128 & 256 \\
\hline$z_{2}$ & 0.003 & $7.952 \mathrm{E}-4$ & $2.031 \mathrm{E}-4$ & $5.075 \mathrm{E}-5$ & $1.269 \mathrm{E}-6$ & $7.927 \mathrm{E}-7$ & $1.982 \mathrm{E}-8$ \\
$p_{2}$ & 2.009 & 1.969 & 2.001 & 2.000 & 2.000 & 2.000 & 2.000 \\
$z_{4}$ & $3.590 \mathrm{E}-5$ & $2.470 \mathrm{E}-6$ & $1.590 \mathrm{E}-7$ & $1.008 \mathrm{E}-8$ & $6.345 \mathrm{E}-10$ & $3.971 \mathrm{E}-11$ & $3.640 \mathrm{E}-13$ \\
$p_{4}$ & 3.8612 & 3.9580 & 3.9797 & 3.9890 & 3.9982 & 3.9990 & \\
$z_{6}$ & $2.506 \mathrm{E}-7$ & $4.132 \mathrm{E}-9$ & $6.755 \mathrm{E}-11$ & $1.069 \mathrm{E}-12$ & $1.338 \mathrm{E}-15$ & & \\
$p_{6}$ & 5.9223 & 5.9345 & 5.9818 & 5.9904 & & & \\
\hline
\end{tabular}

Table 2. Example: the solution $u, b_{l}=1 ; b_{r}=-1$.

\begin{tabular}{cccccccc}
\hline$N$ & 4 & 8 & 16 & 32 & 64 & 128 & 256 \\
\hline$z_{2}$ & 0.001 & $3.448 \mathrm{e}-4$ & $8.632 \mathrm{E}-5$ & $2.159 \mathrm{E}-5$ & $5.404 \mathrm{E}-6$ & $1.351 \mathrm{E}-6$ & $3.378 \mathrm{E}-7$ \\
$p_{2}$ & 2.0215 & 1.9980 & 1.9995 & 1.9982 & 1.9999 & 2.0000 & 2.0000 \\
$z_{4}$ & $2.334 \mathrm{E}-6$ & $1.548 \mathrm{E}-7$ & $1.001 \mathrm{E}-8$ & $6.375 \mathrm{E}-10$ & $4.023 \mathrm{E}-11$ & $2.457 \mathrm{E}-12$ & $1.527 \mathrm{E}-13$ \\
$p_{4}$ & 3.9146 & 3.9506 & 3.9733 & 3.9859 & 4.0333 & 4.0084 & \\
$z_{6}$ & $2.804 \mathrm{e}-7$ & $5.100 \mathrm{e}-9$ & $8.600 \mathrm{e}-11$ & $1.381 \mathrm{e}-12$ & $2.163 \mathrm{e}-14$ & & \\
$p_{6}$ & 5.7808 & 5.8900 & 5.9609 & 5.9965 & & & \\
\hline
\end{tabular}

The numerical solutions were computed on uniform meshes with $N=$ $4,8,16, \ldots, 256$. The maximum norm of the error and an approximate rate of convergence

$$
z_{n}:=\left\|z_{h}^{(n)}\right\|_{\infty}=\max _{0 \leq i \leq N}\left|z^{(n)}\left(x_{i}\right)\right|, h=\frac{1}{N}, \quad p_{n}=\log _{2} \frac{\left\|z_{2 h}^{(n)}\right\|_{\infty}}{\left\|z_{h}^{(n)}\right\|_{\infty}}
$$


Table 3. Example: the flux $w, b_{l}=0 ; b_{r}=-1$.

\begin{tabular}{cccccccc}
\hline$N$ & 4 & 8 & 16 & 32 & 64 & 128 & 256 \\
\hline$z_{2}$ & 0.625 & 0.157 & 0.039 & 0.010 & 0.003 & $6.152 \mathrm{e}-4$ & $1.538 \mathrm{e}-4$ \\
$p_{2}$ & 1.9922 & 1.9963 & 2.0073 & 1.9709 & 2.0228 & 2.0000 & 2.0000 \\
$z_{4}$ & 0.001 & $7.010 \mathrm{e}-5$ & $4.363 \mathrm{e}-6$ & $2.719 \mathrm{e}-7$ & $1.697 \mathrm{e}-8$ & $1.059 \mathrm{e}-9$ & $9.695 \mathrm{e}-12$ \\
$p_{4}$ & 3.9719 & 4.0060 & 4.0041 & 4.0022 & 4.0021 & 4.0003 & \\
$z_{6}$ & $3.210 \mathrm{E}-7$ & $5.732 \mathrm{E}-9$ & $9.602 \mathrm{E}-11$ & $1.509 \mathrm{E}-12$ & $1.872 \mathrm{E}-15$ & & \\
$p_{6}$ & 5.8076 & 5.8996 & 5.9920 & 5.9990 & & & \\
\hline
\end{tabular}

are presented in Tables $1,2,3$ (subscript $\infty$ is omitted in the notation of the norm).

The computations were done on PC Athlon $900 \mathrm{MHz}$ with MATLAB. For the schemes of order 2, 4, 6 the results are contained in Tables 1,2,3 in which the convergence rates are clearly observed.

\section{Conclusions}

In this paper we generalized the classical Marchuk integral identity in order to obtain conservative high-order difference schemes for one-dimensional convection-diffusion interface problems. The local exact equations admit weak formulation analogical to that proposed by Agoshkov [12]. This property can be used for construction of high-order approximations to degenerate equations, parabolic and elliptic problems [11, 12]. Our schemes, in the case $a(x):=\varepsilon^{2} a(x)$ have diagonal domination which is uniform with respect to small parameter $\varepsilon$ and easily can be implemented to singularly perturbed problems [1].

\section{Acknowledgements}

This research was supported by Bulgarian National Science Fund under Project VY-MI-106/2005.

\section{References}

[1] I. Angelova and L. Vulkov. Singularly perturbed differential equations with discontinuous coefficients and concentrated factors. Appl. Math. and Comp., 158, 683 - 701, 2004.

[2] I. Angelova and L. Vulkov. High order difference schemes based on new Marchuck integral identities for one-dimensional interface problems. J. Numer. Math., 13(1), 1 - 18, 2005. 
[3] S.C. Brenner and L.R. Scott. The mathematical theory of finite element methods. 2-nd ed. Berlin, Heidelberg, New York: Springer-Verlag, 2002.

[4] E.C. Gartland. Compact high-oder finite differences for interface problems in one dimension. IMA Journal of Numerical Analysis, 9, $243-360,1989$.

[5] J.L. Gracia, F. Lisbona and C. Clavero. High order -uniform methods for singularly perturbed reaction-diffusion problems. Lect. Notes in Comp. Sci., 1998, 350 - 359, 2001.

[6] H. Kalis. Some heat transfer problems with convection in multilayer media. In: Proc. of the 2nd Intern. Conference 'Finite difference methods: theory and applications', Institute of Mathematics, Minsk-Belarus, 50 - 55, 1998.

[7] M.V. Kutniv. High order accurate three-point difference schemes for systems of second order odes with a monotone operator. Comp. Math. and Math. Phys., 39, 45 - 60, 1999. (in Russian)

[8] Z. Li. An overview of the immersed interface method and its applications. Taiwanes J. Math., 7(1), 1 - 49, 2003.

[9] R.E. Lynch and J.R. Rice. A high order difference method for differential equations. Math. Comp., 34, $333-372,1980$.

[10] G.I. Marchuk. Numerical methods for reactor calculations. Atomizdat, Moscow, 1958. (in Russian)

[11] G.I. Marchuk. Methods of numerical mathematics. Nauka, Moscow, 1981. (in Russian)

[12] G.I. Marchuk and V.I. Agoshkov. Introduction into projection-mesh methods. Nauka, Moscow, 1981. (in Russian)

[13] P. Matus. Monotone schemes of a higher order of accuracy for differential problems with boundary conditions of the second and third kind. Comp. Meth. Appl. Math., 2, 378 - 392, 2002.

[14] K.W. Morton. Numerical solution of convection-diffusion problems. Chapman Hall, London, 1996.

[15] A.A. Samarskii. The theory of difference schemes. Marcel Dekker Inc., New York, 2001.

[16] A.A. Samarskii and V.L. Makarov. On realization of three-point difference scheme for second-order differential equations with piecewise smooth coefficients. J. of Comp. math. math. Phys., 26, 1254 - 1265, 1990.

[17] C.W. Shu. An overview of high order numerical methods for convection dominated pdes. In: Proc. of Ninth Int. Conf. on Hyp. Problems, March 2002, Pasedana. Springer-Verlag, Berlin, Heidelberg, New York, 79 - 88, 2003.

[18] W.F. Spotz. High-order compact finite difference schemes for computational mechanics. Ph.D.Thesis, University of Texas at Austin, 1995.

[19] W. Tongke. High accuracy finite volume element method for two-point boundary value problem of second order ordinary differential equations. Numerical Math., 11(2), 213 - 225, 2003.

[20] R.S. Varga. Matrix iterative analysis. Englewood Cliffs, NJ: Prentice Hall, 1962. 
Aukštos eilè skirtumų schemos konvekcijos-difuzijos sąveikos uždaviniams

I.T. Angelova

Straipsnyje sukonstruotos ir analizuojamos naujos aukštos eilès kompaktinès baigtiniu skirtumų schemos, aproksimuojančios konvekcijos-difuzijos sąveikos uždavinius vienmačiu atveju. Gautos išreikštinès $O\left(h^{2}\right), O\left(h^{4}\right), \ldots$ eilès tikslumo formulès, pagrisstos Marchuko integralinemis tapatybèmis. Išvestos naujos skaitmeninio integravimo kvadratūrinès nurodyto tikslumo formulès tritaškių schemų skaičiavimui. Pateikti skaitiniai eksperimentai, patvirtinantys teorinius rezultatus. 